\title{
MOLECULAR MECHANISMS OF ISCHEMIC-REPERFUSION INJURY DURING LIVER RESECTION FOR COLORECTAL CANCER METASTASES- STUDY PROTOCOL
}

\author{
Marko Gmijović ${ }^{1}$, Ljiljana Jeremić Savić ${ }^{1,2}$, Milica Nestorović ${ }^{1,2}$, Vanja Pecić ${ }^{1}$, Branko Brankovićc, \\ Miodrag Djordjević ${ }^{3}$, Tatjana Jevtović Stoimenov ${ }^{2}$, Marko Stojanović ${ }^{2}$ Milan Radojković ${ }^{1,2}$, Miroslav Stojanović ${ }^{1,2}$ \\ ${ }^{1}$ Clinic for Digestive Surgery, Clinical Center Niš, Niš, Serbia \\ ${ }^{2}$ Faculty of Medicine, University of Niš, Niš, Serbia \\ ${ }^{3}$ Clinic for Endocrine and Breast Surgery, Clinical Center Niš, Niš, Serbia
}

\begin{abstract}
Bleeding during liver resection is a significant threat to the clinical outcome. Portal triad occlusion with complete ischemia of the liver flow of hepatoduodenal ligament is a well-documented, safe and useful way to reduce this problem. Although the technique is efficient in limiting blood loss, there is still controversy concerning potential disadvantages and subsequent reperfusion liver injury. A prospective analysis will include at least 30 patients 18-75 years old, who are undergoing liver resection due to metastases of the colorectal carcinoma. After signing the informed consent, the parameters will be analyzed in three phases, pre-, intra- and postoperatively. During surgery (indicated by a surgical/oncological team of doctors not related to our study) liver tissue samples will be taken of the "healthy liver" (not involved in the tumor process) in which after tissue homogenization, analysis of parameters will be done responsible for the development of liver injury. This research will not affect the clinical practice, course and outcome of the treatment in patients who are included. The study may be useful for future patients who will undergo liver resections. The application of modern research methods with scientific validity of statistical processing of data and the use of appropriate literature, significant data will be obtained about the character, i.e. intensity of damage of the liver tissue in patients undergoing liver resections.
\end{abstract}

Key words: liver resection, ischemia-reperfusion injury, colorectal carcinoma metastases, apoptosis, vascular isolation of liver.

\section{Research Objective}

The latest achievements in liver resection surgery and significant progress in preoperative estimation, preparation and postoperative monitoring and patient care expanded the indications and range of liver resection procedures. The most important problem during hepatectomy is mandatory control of bleeding from the liver parenchyma. Uncontrolled intra- and postoperative bleeding from the resected liver is still the most severe negative prognostic factor which is in direct correlation with immediate postoperative morbidity and mortality $[1,2,3]$. Besides the immediate metabolic and cardiocirculatory consequences of hypovolemia of different degree, secondary negative effects of excessive bleeding during liver resection are increased need for postoperative transfusion of fresh blood, i.e. blood components and derivatives which are proven to be linked to critical coagulopathies and immunity decrease and shorter postoperative disease-free interval in liver malignancies

Correspondence to: Marko Gmijović, M.D.

Clinic for Digestive Surgery, Clinical Center Niš,

48 Zoran Đinđić Blvd., 18000 Niš, Serbia

Phone: +381655665656

E-mail: dr.gmija@gmail.com

Received May $7^{\text {th }}, 2019$
[4,5]. The intent to overcome this key problem resulted during the last three decades of the last century in the development of a range of different methods of vascular control, or vascular liver isolation, which depend on the location of the lesion in the liver. The condition of the liver parenchyma and the general condition of the patient may cause a complete or partial vascular isolation or transitory liver ischemia by occlusion of inflowing and/or outflowing liver blood vessels. Temporary disruption of only the inflow, or of both inflow and outflow of the circulation (vascular exclusion) of the part or the whole liver aim to quickly (but not hastily) conduct anatomically precise and technically correct liver resections in bloodless conditions. Thanks to its advantages, first of all relatively quick and easy application and satisfactory hemostatic results, the most significant and most often used method of vascular isolation of the liver is the Pringle maneuver. The method was inaugurated in 1908 [6] and it includes a complete interruption of the inflowing liver circulation by clamping of portal vain and hepatic artery, i.e. of the whole hepatoduodenal ligament by a vascular clamp, setting the tourniquet or in urgent situations with fingers. However, the disadvantage of all the methods of vascular isolation of the liver, including the Pringle maneuver, is imminent ischemic and then subsequent 
reperfusion parenchymal liver damage. Although the Pringle maneuver is efficient in reducing the blood loss from the resected liver parenchyma, critical hepatic ischemia that this maneuver causes represents a serious limiting problem that must be taken care of, particularly when there is a previous liver disease with significant vulnerability and sensitivity of the organ to hypoxia. Such transitory inflow occlusion causes parenchymal damage which is defined as ischemia-reperfusion injury (IRI). Etiological basis of this injury is a decrease in the level of intracellular ATP due to cellular anoxia which leads to an increase in intracellular $\mathrm{Ca}^{2+}$ ion concentration [7]. Namely, decreased ATP causes a decrease in active transport of $\mathrm{Ca}^{2+}$ ATPase and opening of $\mathrm{Ca}^{2+}$ voltage-dependent canals during depolarization of the cellular membrane which develops due to reduced activity of $\mathrm{Na}^{+} \mathrm{K}^{+}$ATPase because of which there is an increase in the exit of $\mathrm{K}^{+}$ions from cells and the entrance of $\mathrm{Na}^{+}$ions in the cells. As a result, the concentration of intracellular $\mathrm{Ca}_{2}^{+}$increases significantly and leads to the destruction of the cytoskeleton [8-12]. In order to prevent critical ischemic damage, a timely reoxygenation is necessary. At the same time, however, this reperfusion causes reperfusion injury [13-16]. Namely, xanthine-dehydrogenase is converted into xanthine-oxidase and hypoxanthine, obtained from the degradation of adenine-nucleotides, is accumulated in the ischemic organ. With the beginning of the reperfusion of previously ischemic organ great amounts of superoxides are created from previously accumulated hypoxanthine by xanthinoxidase action which leads to the damage of the cytoplasm, proteins and DNA and prevents the reduction of oxygen in the mitochondria [10]. Then, peroxidation of the cytoplasmatic membrane lipids takes place which is composed of two-layered lipids which causes its damage. Considering literature data which show that ischemia reperfusion injury may affect the course and outcome of the treatment in terms of intraoperative bleeding, prolonged hospitalization, increased morbidity and mortality, the study is directed at identifying the molecular mechanisms of apoptosis which are initiated during ischemia and reperfusion in liver tissue $\mathrm{f}$ humans.

\section{Ethical Standards}

During the research, standard methods of preoperative preparation, operative treatment and postoperative monitoring were used. The participants may not have any direct benefit from participating in the study apart from more systematic monitoring and recording the research parameters. The study may be useful to future patients who will undergo liver resection. Since the research does not disrupt the planned liver resection protocol the participants will not be exposed to risks related to the research. The research does not include the use of untested or new medications or the testing of new surgical interventions. The mode of patient treatment included in the research is identical to the treatment of other patients with the same symptoms who were not included in this research and completely the same as the treatment applied with patients operated on at the Digestive Surgery Clinic of the Clinical Center Niš. The patients were chosen as potential participants because of the planned resection of the liver. The patients may freely accept or reject participation in the research. The participation decision will not influence the mode of operative treatment.

We emphasize that participation is voluntary and that at any given moment, at any stage, the consent may be withdrawn by the patient. The data obtained in the research will be available only to the research team because they are confidential. After informing the patients and obtaining signed consent, every patient is given a code number which will be used for storing all the information related to treatment. The conclusions obtained during the research will published and available to public. Confidential data will not be published.

\section{Material and Methods}

Prospective analysis will include at least 30 patients aged 18-75 who are undergoing liver resection due to metastases of the colorectal carcinoma. The patients included in the research has resectable metastases and were not undergoing neoadjuvant chemotherapy. After signing the informed consent, the parameters will be analyzed in three stages, pre-, intra- and postoperatively. Including and excluding criteria are shown in Table 1. During surgery (indicated by surgical/oncological team

Table 1 Inclusion and exclusion criteria

\begin{tabular}{ll}
\hline Inclusion criteria & Exclusion criteria \\
\hline - Confirmed diagnosis of adenocarcinoma of the & - Local recurrence of colorectal cancer \\
colorectum after curative surgery & - Patients with previous liver injury \\
- Resectable CRC liver metastasis & - Patients with prior liver surgery \\
- No evidence of distant metastases & - Patients who in previous surgery underwent portal vein \\
- No prior chemotherapv for metastatic disease & - ligation \\
- Age $>18$ years $<75$ & - HCV or HBV infection \\
- No infections requiring systemic antibiotic treatment & - liver cirrhosis \\
& - Patients with a history of anv arterial thrombotic event \\
& - within the past 6 months, including angina (stable or \\
\hline
\end{tabular}


independent of our research) samples of liver tissue of healthy liver (not involved in tumor process) will be taken in which, after tissue homogenization, an analysis of parameters responsible for the development of ischemia reperfusion injury will be done.

Preoperative parameters: demographic data (age and sex), standard preoperative investigations which examine liver function in all patients included in the research (alkaline phosphatase, AST, ALT, $\gamma \mathrm{GT}$, bilirubin, LDH, albumin, alpha-fetoprotein), as well as the presence of metabolic syndrome (glucose $\geq 6.1 \mathrm{mmol} / \mathrm{L}$; serum triglycerides $\geq 1.7 \mathrm{mmol} / \mathrm{L}$; serum $\mathrm{HDL}$ cholesterol $<1.04 \mathrm{mmol} / \mathrm{L}$; blood pressure $\geq 130 / 85 \mathrm{~mm} \mathrm{Hg}$ ).

Intraoperative parameters: surgical approach, type and resection circumference, intraoperative blood loss, intraoperative blood compensation, liquid compensation and duration of surgery. During surgery, samples of liver tissue will be taken from the resection area. Liver tissue samples represent the tested groups. Three groups will be formed (I - control group, II - tissue samples during ischemia, III - samples after tissue reperfusion) further divided into seven subgroups based on the time the sample was taken. At zero minute, one sample is taken (control group), then after ischemia of hepatoduodenal ligament (group II) samples are taken within subgroups at 5, 10 and 15 minutes of ischemia. After ischemia, samples are taken (group III) within subgroups at 1, 3 and 5 minutes (Diagram 1). All samples will be stored in special containers and frozen at $-80^{\circ} \mathrm{C}$. The tissue sample and the patient are given a code number. The research will be conducted at the Laboratory for Functional Genomics and Proteomics of the Scientific-Research Center of the Faculty of Medicine, University of Niš. In order to estimate the biochemical parameters of programmed cell death the liver tissue was first cut into small pieces and then finely cut in ice cold water with a homogenization apparatus. The homogenate $(10 \% \mathrm{v} / \mathrm{v})$ will be centrifuged at 1500 rpm for 10 minutes at $4^{0} \mathrm{C}$. The activity of caspase -3 , acidic DNaseand Bax protein concentration will be determined in the supernatant. Caspase-3 will be determined with commercial ELISA kit (Cusabio Technology LLC, USA). The activity of acidic DNase (DNase-2) will be measured using the method of Bartholeyns et al., (1975) and the formed acid-soluble nucleotides were measured spectrophotometrically. Enzymes activity was expressed in international units in gram of protein (U/g protein). The concentration of Bax protein in liver homogenate will be determined with a commercial ELISA kit (Human Apoptosis regulator BAX ELISA Kit, cusabio Technology LLC, USA). The activity of the enzymes of alkaline phosphatase (AF), aspartate aminotransferase (AST), alanine aminotransferase (ALT), gamma-glutamyl transferase (GGT) and lactate dehydrogenase (LDH) in serum will be determined with commercial Sigma tests (St. Louis, MO, USA) using the spectrophotometric method at the Central Laboratory of the Clinical Center Niš.

Postoperative parameters: The postoperative liver function as well as the presence of metabolic stress will be monitored by analyzing patients' blood samples at the Central Laboratory of the Clinical Center Niš as part of control postoperative monitoring.

\section{The Aim of the Research}

The research deals with problems accompanying the occlusion of the portal triad and tissue damage at molecular level in patients who need some of the liver resections in terms of ischemia during resection and later liver tissue reperfusion.

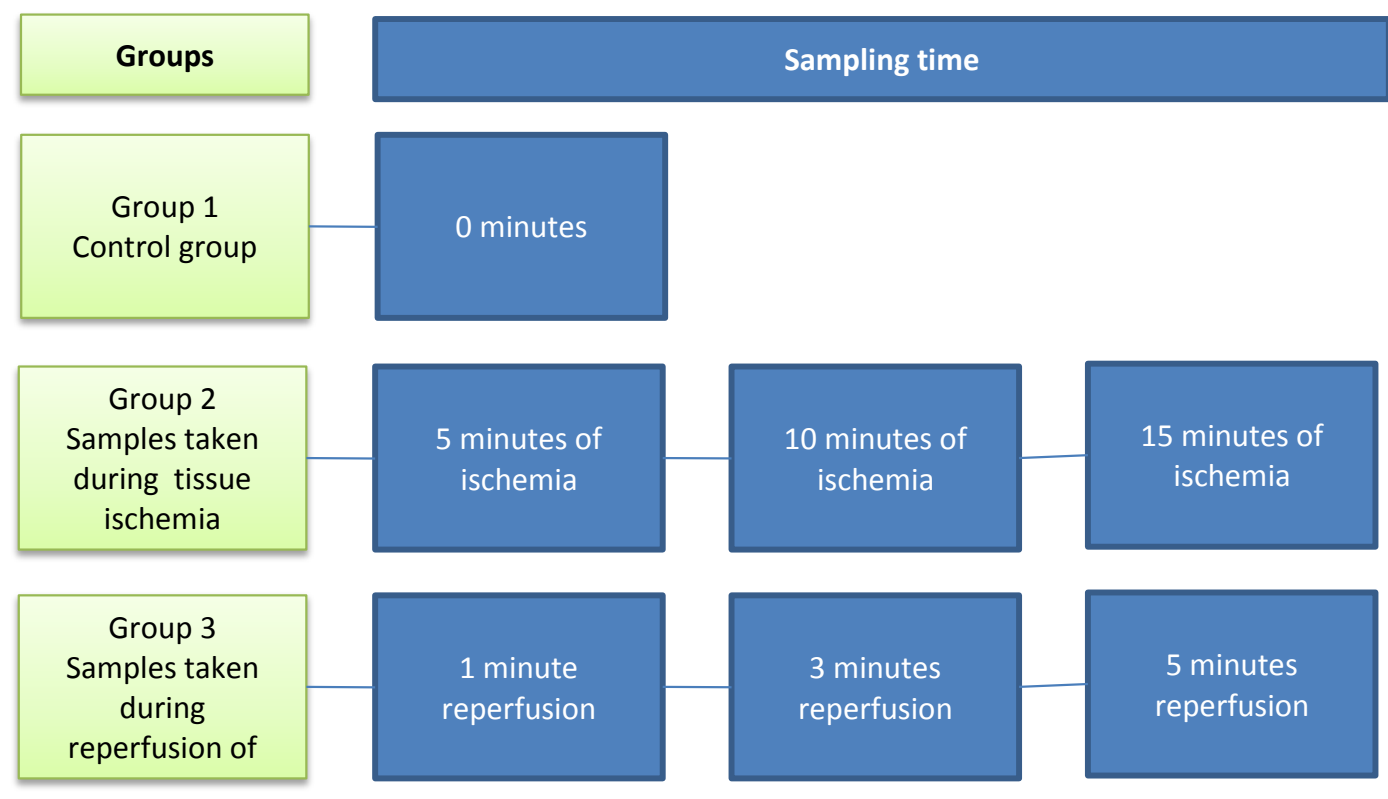

Diagram 1 Intraoperative sampling of the liver tissue 
End points:

1. Determine the degree of apoptosis of the liver parenchyma intraoperatively during ischemia and during tissue reperfusion in specific time intervals (the activity of caspase-3 and deoxyribonuclease-II will be determined as well as the level of Bax protein in liver tissue).

2. Determine preoperatively and postoperatively the condition of liver parenchyma by determining the biochemical parameters of the liver function (the activity of the alkaline phosphatase enzyme, AST, ALT, $\gamma$ GT,biliubin, LDH, albumin, alpha-fetoprotein, urea) and the parameters of metabolic syndrome (glucose, serum triglycerides, serum HDL cholesterol, blood pressure)

3. Determine the correlation of apoptosis parameters with functional tests of the liver during ischemia and reperfusion.

\section{Statistical Analysis}

The estimation was done by double testing of the null hypothesis with independent t-test with accepted values of type I error $\alpha=0,05$ and power of the study $90 \%$. The total study sample is 30 participants. Sample size was calculated in $\mathrm{G}$ power program 3.1.9.2. On the basis of the gathered data, a database will be created in software package SPSS 20.0 which will be used for further statistical analysis. Statistical analysis of data will include the methods of descriptive and analytical statistics. The results will be represented in tables and graphs. All attributive characteristics of the monitoring are described in absolute and relative numbers. The description of numerical forms of monitoring we will use the measures of averages (median and arithmetic mean) and standard deviation. The choice of tests used for numeric observations depend on the nature of the distribution of the numeric markings of observation and group number. Normal distribution will be tested using KolmogorovSmirnov test. The testing of the hypothesis of equality of mean values in cases when two groups exist will be done by t-test for dependent or independent samples in case of normal distribution and Wicoxon test for dependent and Mann-Whitney test for independent samples when distribution is nor normal. If three groups exist, ANOVA (one-way analysis of variance) test will be used for normal distribution and Kruskal-Wallis test for other than normal distribution of data. For correlation analysis, Pearson correlation coefficient will be used with normal distribution or Spearman coefficient correlation when distribution is not normal. For determining the link between the tested parameters and the genotypes of the tested polymorphism univariate and multivariate logistic regression analysis will be used. Statistical hypothesis will be tested on the level of significance for risk of $\alpha=0.05$. i.e. the difference between the samples will be considered significant at $\mathrm{p}<0.05$.

\section{Discussion}

The liver is an organ which contains the most macrophages compared to other organs in the body and it has the ability to secrete oxygen radicals and tissue toxic mediators. This biological characteristic of the liver may lead to higher sensitivity. i.e. susceptibility to ischemic damage. The examination of the ischemic-reperfusion injury was done mostly on experimental models. In the last two decades of the last century the accumulated evidence has shown that the ischemic reperfusion injury of the liver is induced or potentiated by the action i.e. metabolism of several toxic chemical mediators such as reactive (free) oxygen radicals, proteases (elastase), inflammatory cytokines, thrombocyte-activating factor, vasoconstrictors endothelin and thromboxane etc., i.e. by pathophysiological events in which they participate (neutrophil infiltration and their adhesion to hepatic sinusoidal endothelial cells, elastase hyperproduction, sinusoid constriction and microcirculation damage, apoptosis, degradation of phospholipid cell membrane etc. $[9,15]$. On the basis of these pathophysiological mechanisms of ischemia reperfusion injury numerous strategies have been suggested for its prophylaxis and treatment the majority of which are based on the inhibition of negative effect mostly of oxygen radicals, inflammatory toxins and adhesion molecules and cascading processes in which they participate [17]. The edema of sinusoidal and Kupfer cells after ATP depletion during liver ischemia, increased production of strong endothelin vasoconstrictor and probably decreased NO production are pathophysiological mechanisms which contribute to the reduction of sinusoidal lumen [10]. Apoptosis or programmed cell death has a key role in many biological processes. Its determination is highly significant in various fields of modern medicine. In this case it represents the beginning of hepatocyte damage which leads to permanent damage. In order to determine the degree of programmed cell death, we can use markers such as caspase-3 - enzymes which initiate the apoptosis process, acidic DNase (DNase-2) which is the final stage of apoptosis - it disintegrates the nucleus material and BAX protein which points to the mitochondrial pathway of apoptosis activation. Since the effects of ischemia and reperfusion are the initiators of apoptosis which has several levels - we have chosen these parameters in order to determine the degree of programmed cell death. Based on the level of apoptosis we can conclude whether the patients should be administered apoptosis inhibitors to prevent cell death. So far, the researches in ischemia reperfusion injury mechanism were done mostly on experimental models which included working on lab animals (ex hominem) or tissue cultural (ex situ or in vitro examinations). In addition, clinical and experimental investigations are ethically inacceptable or technically undoable in the majority of conditions and diseases which cause this syndrome, except determining some parameters from systematic or local circulation, in other words-it is impossible to de- 
termine them in the tissue involved with ischemiareperfusion injury. Therefore, we believe that the most precise examination of injury mechanisms can be achieved by analyzing the listed parameters from liver tissue homogenate collected during resection intervention on the liver. Standard clinical liver resection procedure during which temporary ischemia and subsequent reperfusion are induced have enabled developing a unique experimental model during which samples are taken 'in situ' and 'in vivo' from "healthy liver" (not involved in the tumor process) in which after tissue homogenization the parameters responsible for the development of ischemia reperfusion injury is done.

\section{Conclusion}

The significant progress in liver resection techniques as well as new treatment methods have brought about improvement in the quality of life and longer survival of patients with liver damage who need some of the resection procedures in treatment. However, contrary to these favoring factors, resection procedures carry the risk of liver tissue damage which in many cases many jeopardize patient's life.

Having in mind the small number of such and similar experimental studies, the application of modern research methods, with scientific validity of statistical data analysis and the use of literature, it is expected that significant data about the character i.e. the intensity of liver tissue injury in patients during resection procedures of the liver will be obtained.

10. Serracino-Inglott F, Habib NA Mathie RT. Hepatic ischemiareperfusion injury. Am J Surg 2001; 181:160-166.

11. Bilzer M, Gerbes AL. Preservation injury of the liver:Mechanisms and novel therapeutic strategies. J Hepatol 2000; 32:508-515.

12. Zhong Z, Ramshesh VK, Rehman H, et al. Activation of the oxygen-sensing signal cascade prevents mitochondrial injury after mouse liver ischemia-reperfusion. Am J Physiol Gastrointest Liver Physiol 2008; 95:G823-832.

13. Theruvath TP, Snoddy MC, Zhong Z, et al. Mitochondrial permeability transition in liver ischemia and reperfusion: role of cJun N-terminal kinase 2. Transplantation 2008; 85:1500-1504.

14. Wilson GC, Kuboki S, Freeman CM, et al. CXC chemokines function as a rheostat for hepatocyte proliferation and liver regeneration. PLoS One 2015; 10(3):e0120092

15. Boulter L, Lu WY, Forbes SJ. Differentiation of progenitors in the liver: A matter of local choice. J Clin Invest 2013; 123(5):18671873.

16. Konishi T, Lentsch AB. Hepatic Ischemia/Reperfusion: Mechanisms of Tissue Injury, Repair, and Regeneration. Gene Expr 2017; 17(4):277-287.

17. Jeon J, Watkins A, Wagener G, et al. Complex hepatectomy under total vascular exclusion of the liver: impact of ischemic preconditioning on clinical outcomes. World J Surg 2013; 37(4):838-469. 